\title{
Deep spectral learning for label-free optical imaging oximetry with uncertainty quantification
}

\author{
Rongrong Liu', Shiyi Cheng ${ }^{2}$, Lei Tian $\mathbb{1}^{2}$ and Ji Yi²,3,4
}

\begin{abstract}
Measurement of blood oxygen saturation $\left(\mathrm{s}_{2}\right)$ by optical imaging oximetry provides invaluable insight into local tissue functions and metabolism. Despite different embodiments and modalities, all label-free optical-imaging oximetry techniques utilize the same principle of $\mathrm{s}_{2}$-dependent spectral contrast from haemoglobin. Traditional approaches for quantifying $\mathrm{s}_{2}$ often rely on analytical models that are fitted by the spectral measurements. These approaches in practice suffer from uncertainties due to biological variability, tissue geometry, light scattering, systemic spectral bias, and variations in the experimental conditions. Here, we propose a new data-driven approach, termed deep spectral learning (DSL), to achieve oximetry that is highly robust to experimental variations and, more importantly, able to provide uncertainty quantification for each $\mathrm{sO}_{2}$ prediction. To demonstrate the robustness and generalizability of DSL, we analyse data from two visible light optical coherence tomography (vis-OCT) setups across two separate in vivo experiments on rat retinas. Predictions made by DSL are highly adaptive to experimental variabilities as well as the depth-dependent backscattering spectra. Two neural-network-based models are tested and compared with the traditional least-squares fitting (LSF) method. The DSL-predicted $\mathrm{sO}_{2}$ shows significantly lower mean-square errors than those of the LSF. For the first time, we have demonstrated en face maps of retinal oximetry along with a pixel-wise confidence assessment. Our DSL overcomes several limitations of traditional approaches and provides a more flexible, robust, and reliable deep learning approach for in vivo non-invasive label-free optical oximetry.
\end{abstract}

\section{Introduction}

Microvascular systems deliver oxygen to support cellular metabolism and maintain biological functions. Within the local microenvironment of blood vessels, oxygen unloads from haemoglobin and diffuses freely from red blood cells (RBCs) to tissues following the gradient of oxygen partial pressure $\left(\mathrm{pO}_{2}\right)$, which determines the oxygen saturation $\left(s \mathrm{O}_{2}\right)$ of haemoglobin. The measurement of microvascular $s \mathrm{O}_{2}$ can thus help in assessing the local tissue oxygenation and provide invaluable insight into local tissue metabolism, inflammation, and oxygenrelated pathologies. It can also offer diagnosis and

Correspondence: Ji Yi (jiyi@bu.edu)

${ }^{1}$ Department of Biomedical Engineering, Northwestern University, Evanston, IL 60208, USA

${ }^{2}$ Department of Electrical and Computer Engineering, Boston University, Boston, MA 02215, USA

Full list of author information is available at the end of the article. prognosis for several major diseases, such as cancers, diabetic milieu and complications, cardiovascular diseases, dementia, etc ${ }^{1-5}$.

In recent years, several non-invasive and label-free optical-imaging oximetry techniques have been developed to measure microvascular $s \mathrm{O}_{2}$. Despite their differences, the fundamental mechanism is the same, being based on the $s \mathrm{O}_{2}$-dependent spectral contrast from haemoglobin ${ }^{6}$. The spectral measurement is then related to $s \mathrm{O}_{2}$ through a complex physical model incorporating tissue geometry, heterogeneous tissue scattering, light attenuation and propagation, and imaging optical instruments. This model is often simplified and analytically formulated under different approximations and assumptions. Examples include spatial frequency domain imaging ${ }^{7,8}$ in the diffusive regime under the P3 approximation, multiwavelength imaging ${ }^{9-12}$ and visible light optical coherence tomography (vis-OCT) ${ }^{13-15}$ in the ballistic regime

\section{(c) The Author(s) 2019}

(c) (i) Open Access This article is licensed under a Creative Commons Attribution 4.0 International License, which permits use, sharing, adaptation, distribution and reproduction cc) in any medium or format, as long as you give appropriate credit to the original author(s) and the source, provide a link to the Creative Commons license, and indicate if changes were made. The images or other third party material in this article are included in the article's Creative Commons license, unless indicated otherwise in a credit line to the material. If material is not included in the article's Creative Commons license and your intended use is not permitted by statutory regulation or exceeds the permitted use, you will need to obtain permission directly from the copyright holder. To view a copy of this license, visit http://creativecommons.org/licenses/by/4.0/. 
based on Beer's law combined with the first Born approximation ${ }^{16-25}$, photoacoustic microscopy/tomography assuming a uniform laser fluence inside the tissue $^{26,27}$, and photothermal imaging assuming a linear relation between the blood absorption and the change in the optical signal ${ }^{28-31}$. The $s \mathrm{O}_{2}$ estimation thus requires solving an ill-posed inverse problem that is inevitably subject to model inaccuracies, noise, systemic spectral bias, and experimental conditions. One widely used inversion method is the spectral least-squares fitting (LSF), which estimates the $s \mathrm{O}_{2}$ by matching the spectral data with the analytical model, as shown in Fig. 1a. However, multiple sources of spectral errors exist in practice that are impossible to fully parameterize in an analytical form, which in turn compromises the $s \mathrm{O}_{2}$ estimation accuracy, repeatability, and cross-comparison between different devices, test subjects, and time. Therefore, it is imperative to develop a more robust model to enable more accurate quantification of microvascular $s \mathrm{O}_{2}$ for label-free optical-imaging oximetry.

In this work, we develop a new data-driven deep spectral learning (DSL) method to enable highly robust and reliable $s \mathrm{O}_{2}$ estimation, as shown in Fig. 1b. By training a neural network to directly relate the spectral measurements to the corresponding independent $s \mathrm{O}_{2}$ labels, DSL bypasses the need for a rigid parametric model, similar to existing deep-learning methods for solving optical inverse problems ${ }^{32-36}$. We show that DSL can be trained to be highly robust to multiple sources of variabilities in the experiments, including different setups, imaging protocols, speeds, and other possible longitudinal variations.

An essential feature of our DSL method is uncertainty quantification. Due to biological variations and tissue heterogeneity, an assessment of the reliability of each $s \mathrm{O}_{2}$ measurement is crucial in clinical applications and for guarding against vulnerabilities in making overly confident predictions when imaging rare cases ${ }^{37}$. Existing modelbased methods generate a single value of $s \mathrm{O}_{2}$ for each spectral measurement, i.e., a point estimate. The accuracy and uncertainty of the point estimate can be assessed only by taking repeated measurements against the ground truth in a well-controlled experiment. This uncertainty estimation presents a clear limitation for many biomedical applications in which the ground truth is often inaccessible in vivo, and the statistical analysis can be performed only retrospectively on those repeated measurements ${ }^{13,15,18,31,38}$. Instead of assessing the variabilities in the data retrospectively, we develop our DSL model based on an uncertainty learning framework ${ }^{36}$ to encapsulate the statistics in the learned model, essentially shifting the burden of repeated measurements in the model-based methods to the training phase of DSL. After the training, the DSL model predicts both $s \mathrm{O}_{2}$ and its tandem standard deviation, assessing the uncertainty for each $s \mathrm{O}_{2}$ prediction (i.e., a statistical distribution describing all possible $s \mathrm{O}_{2}$ levels of each prediction given the measurements).

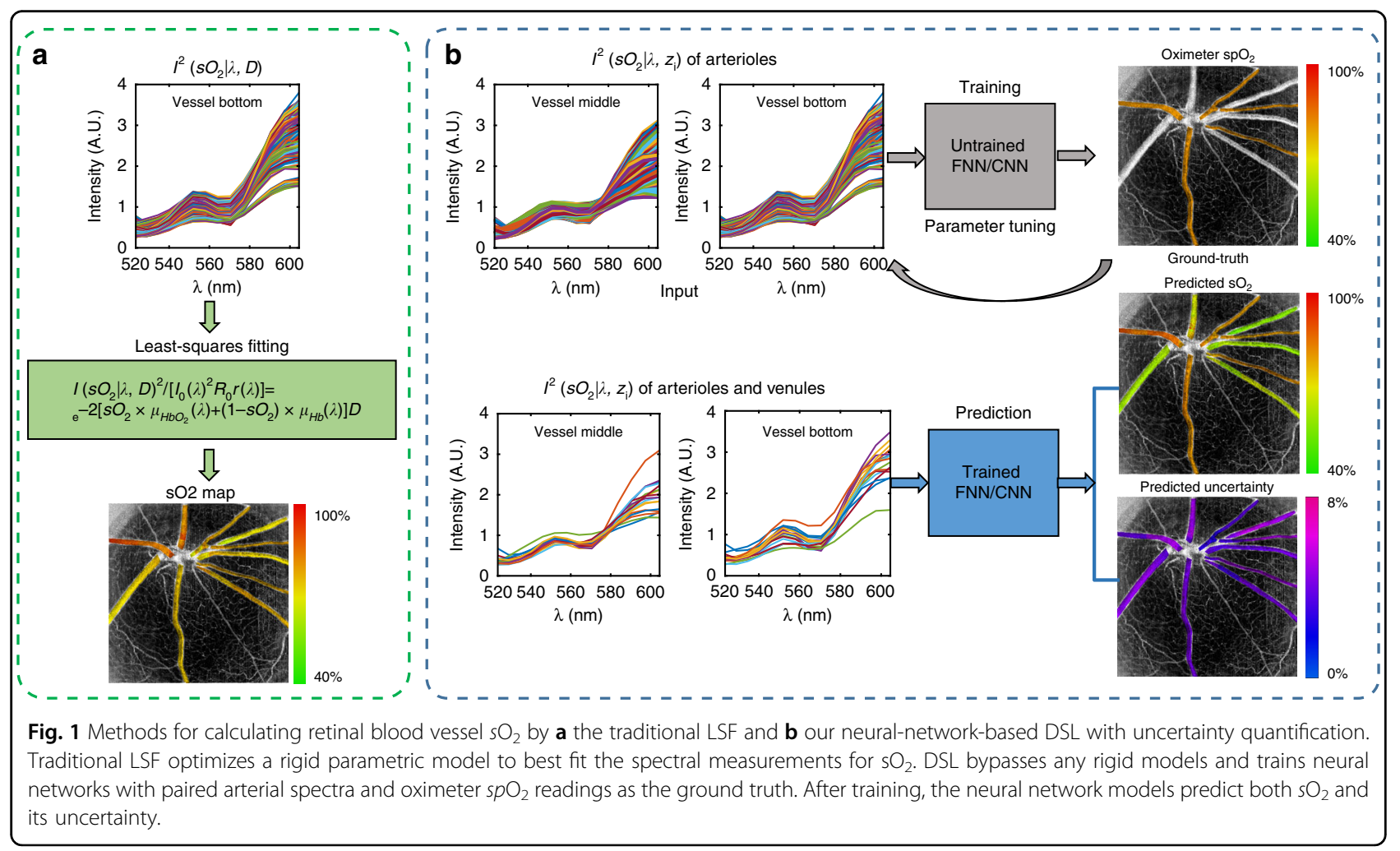


Most importantly, we show that the DSL-predicted statistics closely match those obtained from ensemble calculations. This means that the confidence level calculated from the DSL prediction can be used as a surrogate estimate to the true accuracy of the estimate, making DSL reliable.

We demonstrate DSL using two sets of vis-OCT experiments for oximetry on rat retinas from refs. ${ }^{13,15}$. Vis-OCT in rodent and human retinas has been extensively demonstrated in recent years ${ }^{19,39-42}$, and several studies have shown significant clinical potential in the diagnosis and prognosis of several major retinal diseases using oximetry ${ }^{43-47}$. Two DSL models are investigated, including a 1D fully connected neural network (FNN) and a 1D convolutional neural network $(\mathrm{CNN})$, the network architectures of which are shown in Fig. 2a, b, respectively. Our results show that both DSL models significantly outperform the LSF, in terms of both the estimation accuracy and the robustness to experimental variations. We further conduct a quantitative statistical analysis based on uncertainty learning to establish the confidence level of the two proposed models and further justify the reliability of DSL. Finally, imaging oximetry is demonstrated on en face $s \mathrm{O}_{2}$ maps of rat retinas along with the corresponding uncertainty maps, providing a visualization of the DSL predictions. This process allows us to assess the accuracy of the prediction based on the underlying physiological conditions.

\section{Results}

\section{Data source}

To evaluate the effectiveness of the DSL approach, we compiled two datasets from the previous literature on vis-
OCT retinal oximetry ${ }^{13,15}$. Specifically, the data in Fig. 3a are from ref. ${ }^{13}$, and that in Fig. 3b, from ref. ${ }^{15}$. Both datasets used similar experimental protocols in which the oxygen content in the ventilation gas was adjusted to induce systemic hypoxia or hyperoxia, and vis-OCT measurements were taken under each ventilation condition. In ref. ${ }^{13}$, four rats were measured under six ventilation conditions, from normoxia $\left(21 \% \mathrm{O}_{2}, 79 \% \mathrm{~N}_{2}\right)$, to five hypoxia challenges, in which the oxygen content was reduced step-wise: step $1\left(19 \% \mathrm{O}_{2}, 81 \% \mathrm{~N}_{2}\right)$, step $2(17 \%$ $\left.\mathrm{O}_{2}, 83 \% \mathrm{~N}_{2}\right)$, step $3\left(15 \% \mathrm{O}_{2}, 85 \% \mathrm{~N}_{2}\right)$, step $4\left(13 \% \mathrm{O}_{2}\right.$, $\left.87 \% \mathrm{~N}_{2}\right)$, and step $5\left(9 \% \mathrm{O}_{2}, 91 \% \mathrm{~N}_{2}\right)$. In ref. ${ }^{15}$, eight rats were measured under five ventilation conditions, sequencing from normoxia, hyperoxia $\left(100 \% \mathrm{O}_{2}\right)$, 5\% carbon dioxide $\left(21 \% \mathrm{O}_{2}, 74 \% \mathrm{~N}_{2}, 5 \% \mathrm{CO}_{2}\right)$, hypoxia $(10 \%$ $\mathrm{O}_{2}, 90 \% \mathrm{~N}_{2}$ ), and finally to normoxia. Under each ventilation condition, the systemic arterial $s p \mathrm{O}_{2}$ reading was taken by a pulse oximeter attached to a rear leg of each rat. All the vis-OCT and pulse oximetry measurements were taken approximately one minute after the ventilation transition, when the $s p \mathrm{O}_{2}$ readings were stable. The $s p \mathrm{O}_{2}$ readings are used as the ground truth label for the major retinal arterioles for neural network training. Depthdependent backscattering spectra of rat retinal arterioles in vis-OCT were extracted under each ventilation condition as spectral input to the neural network. The extracted arteriole spectra with the $\mathrm{spO}_{2}$ labels were then split into training and testing sets. In ref. ${ }^{13}$, data from three rats were used as the training sets, with the remaining one as the testing set. In ref. ${ }^{15}$, data from seven rats were used as the training sets, and the remaining one was the testing set. We mixed the training data from both

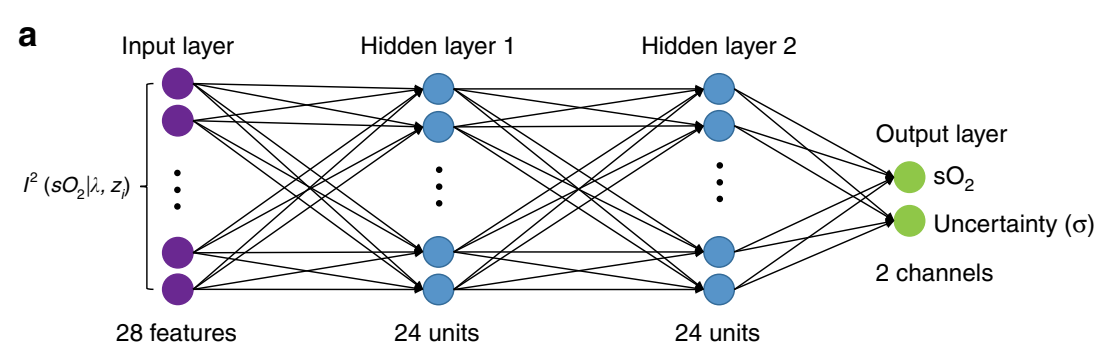

b
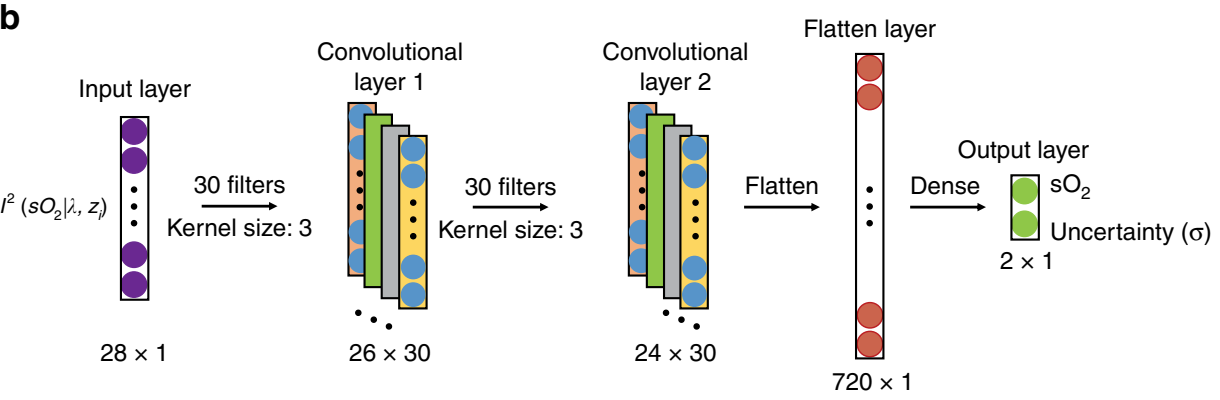

Fig. 2 Structures of the FNN model $\mathbf{a}$ and the $\mathrm{CNN}$ model $\mathbf{b}$ for $\mathrm{sO}_{2}$ prediction, with uncertainty quantified by the predicted standard deviation $\sigma$. 
studies to train the DSL models and make predictions on the testing data from both studies. All the training/testing and the subsequent data analysis are identical for CNN and FNN to compare our DSL approach with the standard LSF. Figure $3 \mathrm{a}, \mathrm{b}$ summarize the number of spectra extracted and the corresponding $s p \mathrm{O}_{2}$ labels in descending order, respectively. Figure 3c shows the histogram of all the training and testing spectra with their corresponding $s p \mathrm{O}_{2}$ labels.

\section{Prediction of arterial $\mathrm{sO}_{2}$}

Once the networks are trained, the $s \mathrm{O}_{2}$ predictions from the testing set obtained by a FNN and CNN are plotted in Fig. $4 \mathrm{a}, \mathrm{b}$, respectively, along with the ground truth oximeter $s p \mathrm{O}_{2}$ readings and the LSF estimates for comparison. Our
DSL models were trained by a combination of both datasets from refs. ${ }^{13,15}$. We optimized the LSF model parameters on the same data pool to ensure a fair comparison between the two methods. The detailed descriptions of the LSF model and parameter optimization are provided in the "Materials and methods" section. The first 248 testing spectra are from ref. ${ }^{13}$, and the remaining 254 are from ref. ${ }^{15}$. In general, the $s \mathrm{O}_{2}$ estimations made using both DSL models (FNN and $\mathrm{CNN}$ ) and LSF agree with the $s p \mathrm{O}_{2}$ readings. A closer look reveals that DSL predictions have lower variations than that of LSF, which we attribute to the DSL's improved robustness to noise and other random signal fluctuations. The absolute errors between the $s \mathrm{O}_{2}$ predictions and the corresponding $s p \mathrm{O}_{2}$ are plotted in Fig. $4 \mathrm{c}, \mathrm{d}$, respectively. Errors from both DSL models are significantly lower than
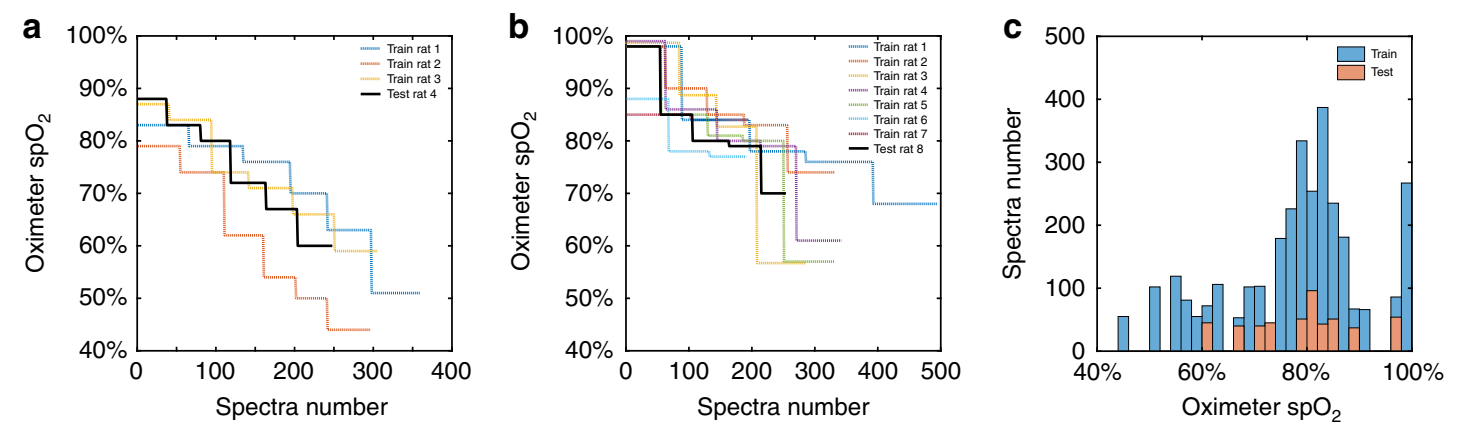

Fig. 3 Oximeter $\mathrm{spO}_{2}$ readings (ground truth labels) for training and testing. a The readings of rat retinal arterioles from normoxia to hypoxia from ref. ${ }^{13}$. $\mathbf{b}$ The readings of rat retinal arterioles from hyperoxia, $5 \% \mathrm{CO}_{2}$, normoxia to hypoxia from ref. ${ }^{15}$. $\mathbf{c}$ Histograms of all $s p \mathrm{O}_{2}$ readings for both the training and testing sets.

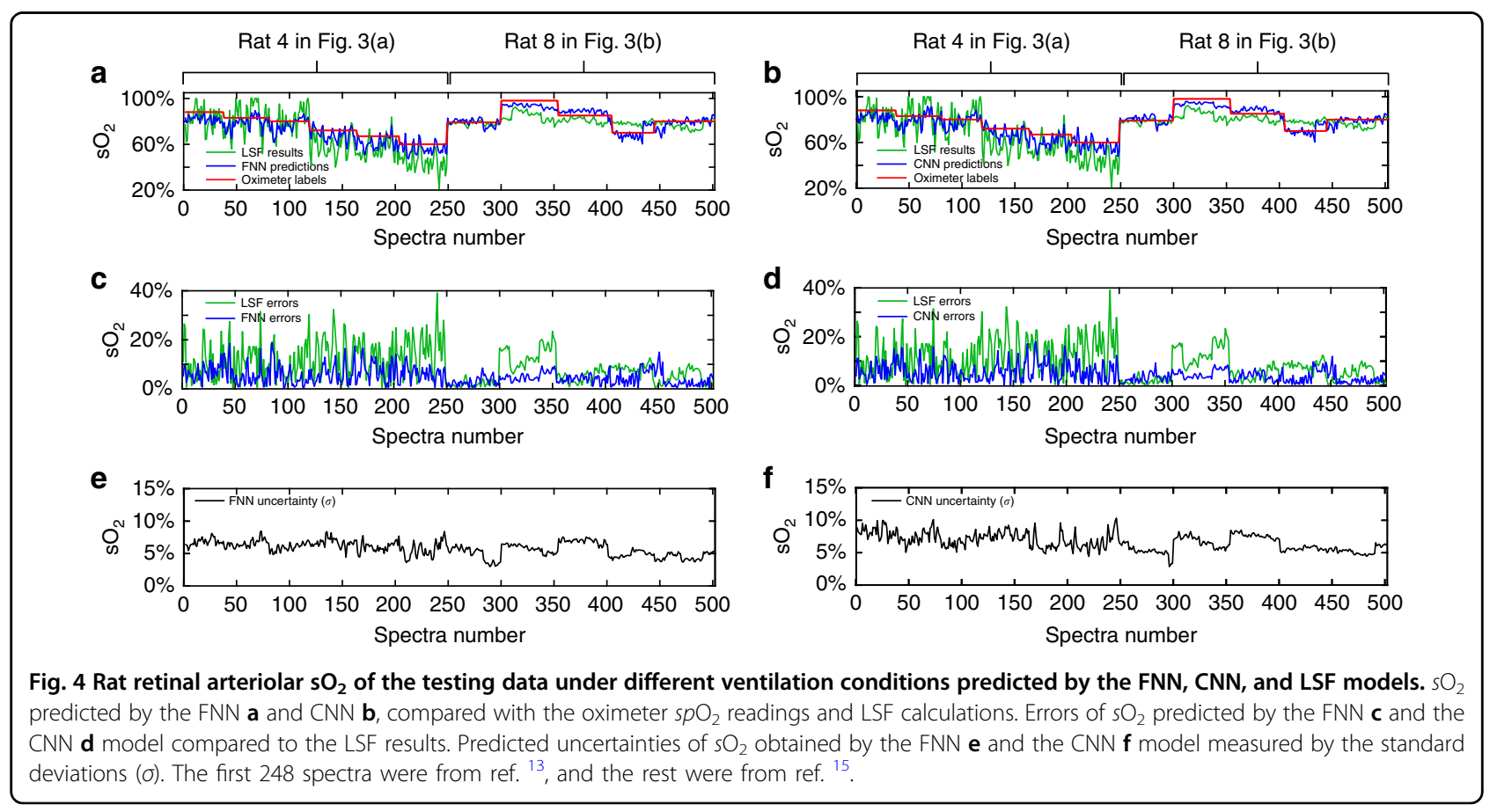


that of the LSF model. To quantitatively compare the three different models, we calculate the mean square errors (MSEs) of the FNN and CNN models to be $0.3539 \times 10^{-2}$ and $0.3200 \times 10^{-2}$, respectively, both of which are $<1 / 3$ the MSE from LSF $\left(1.358 \times 10^{-2}\right)$. An important feature of our DSL models is its ability to quantify uncertainty via the tandem standard deviation $(\sigma)$ for each $s \mathrm{O}_{2}$ prediction (Fig. $4 \mathrm{e}, \mathrm{f})$. Overall, both FNN and CNN predict $\sigma$ to be $\sim 5-7 \%$, and the variation in $\sigma$ justifies the use of a heterogeneous model in our customized loss function. We also see that the prediction for ref. ${ }^{15}$ has slightly lower variation in $\sigma$ than that in ref. ${ }^{13}$, presumably due to higher animal numbers and larger training datasets (Fig. 3). Out of the 2779 training spectra, 1930 are from ref. ${ }^{15}$.

To demonstrate the generality of our DSL approach, we trained DSL and optimized LSF for each of the two datasets separately and repeated the above comparisons. The accuracies of the two DSL models are consistently better than that of LSF (Figs. S1 and S2) by approximately three times (Table S1). Even when we used data from one reference for training/optimization and tested models on the data from the other reference, DSL still outperformed LSF by more than four times (Fig. S3, Table S1). Interestingly, the uncertainty estimated by DSL increases significantly to $\sim 10 \%$ when the networks are tested on a completely new data source (Fig. S3), truthfully reflecting the lower confidence levels of the two networks in this situation.

\section{Evaluation of the quantified uncertainty}

Our uncertainty quantification assumes that the predicted $\mathrm{sO}_{2}$ follows a heterogeneous Gaussian distribution given different spectral inputs. To validate our uncertainty metrics, we retrospectively calculated the actual probability that the ground truth $\left(s p \mathrm{O}_{2}\right)$ falls within a certain confidence interval of the predicted $\mathrm{sO}_{2}$ and summarize the results using the reliability diagram ${ }^{33,36,48}$. To construct a reliability diagram, we gather a sub-set of predictions with a specific standard deviation $\sigma_{0}$. We then calculate the probability from this sub-set of data that satisfy the criterion of $\left|\left[s \mathrm{O}_{2}\right]_{i}-\left[s p \mathrm{O}_{2}\right]_{i}\right|<\eta \sigma_{0}$, where $\left[s \mathrm{O}_{2}\right]_{i}$ and $\left[s p \mathrm{O}_{2}\right]_{i}$ are the prediction and the corresponding ground truth from the $i$ th vis-OCT spectrum, respectively:

$$
P\left(\sigma_{0}, \eta\right)=\frac{1}{\left|S_{\sigma_{0}}\right|} \sum_{i \in S_{\sigma_{0}}} \mathrm{I}_{\left\{\left|\left[\mathrm{O}_{2}\right]_{i}-\left[s p \mathrm{O}_{2}\right]_{i}\right|<\eta \sigma_{0}\right\}}
$$

where $\eta$ is a variable that defines the confidence interval and $S$ denotes the sub-set of the prediction with the specified standard deviation. In practice, we relaxed $\sigma_{0}$ to $\sigma_{0} \pm 1 \%$ to include sufficient data to ensure reliable statistical calculations. Intuitively, the probability will approach 1 when $\eta$ increases, i.e., a larger error tolerance.
At the same time, $\eta$ has a one-to-one correspondence to the theoretical confidence value calculated from the normal distribution. The reliability diagram essentially plots the actual probability against $\eta$ or the theoretical confidence. For an ideal well-calibrated model, the actual probability $P\left(\sigma_{0}, \eta\right)$ should equal the theoretical confidence-falling on the diagonal line in the graph. When the actual probability $P\left(\sigma_{0}, \eta\right)$ is lower than the theoretical confidence, it indicates that the model is over-confidentthe reliability curve is under the diagonal line. When the actual probability $P\left(\sigma_{0}, \eta\right)$ is higher than the theoretical confidence, it indicates that the model is conservativethe reliability curve is above the diagonal line.

The reliability diagrams for both models are shown in Fig. 5. To cover over $90 \%$ of the total 502 predictions of the testing data in the reliability diagram, we set $\sigma_{0}=5 \%$ and $7 \%$ for the FNN model and $\sigma_{0}=5 \%, 7 \%$ and $9 \%$ for the $\mathrm{CNN}$ model. For both models, the $s \mathrm{O}_{2}$ predictions with uncertainty falling within the $7 \pm 1 \%$ range and higher are slightly conservative, with $P\left(\sigma_{0}, \eta\right)$ higher than the predicted confidence; for the FNN model, the $s \mathrm{O}_{2}$ predictions with uncertainty $\sigma_{0}=5 \%$ are slightly overconfident since $P\left(\sigma_{0}, \eta\right)$ is lower than the confidence (Fig. $5 \mathrm{a}$ ); while for the CNN model, the results falling within $5 \pm 1 \%$ are quite close to the diagonal line (Fig. 5b), indicating a well-calibrated DSL model in this regime. In ref. ${ }^{13}$, the accuracy for $s \mathrm{O}_{2}$ calculated by LSF was estimated to be within $\pm 4 \%$ relative error in a well-controlled in vitro blood calibration experiment, using blood analyser readings as the ground truth. The uncertainty predicted here $\left(\sim 5 \% s \mathrm{O}_{2}\right)$ by DSL agrees reasonably well with the in vitro calibration result.

Figure S4 illustrates the sub-sets of data when $\sigma_{0}=5 \%$, $7 \%$ and $9 \%$ for both models. Table 1 provides a summary of the fitting parameters, slope, and constant and how many of the total 502 testing data points were counted for by the statistical analysis within each uncertainty range.

\section{En face $\mathrm{sO}_{2}$ maps with uncertainty quantification}

After model testing and uncertainty analysis, we used the testing data from ref. ${ }^{15}$ and applied the FNN and $\mathrm{CNN}$ models for retinal-imaging oximetry in comparison to LSF (Fig. 6) under three different ventilation conditions. The oximetry obtained by both the FNN and CNN clearly reflects the $s \mathrm{O}_{2}$ changes of all vessels from hypoxia, normoxia, to hyperoxia, with the predicted $s \mathrm{O}_{2}$ of arterioles matching the oximeter $s p \mathrm{O}_{2}$ readings well. The $s \mathrm{O}_{2}$ contrast between arteries and veins is also clearly visualized in hypoxia and normoxia. In comparison, the $s \mathrm{O}_{2}$ predicted by the LSF has less arteriovenous contrast, and the $s \mathrm{O}_{2}$ changes with increasing ventilation oxygen level are not as significant as those identified by DSL. The estimated arterial $s \mathrm{O}_{2}$ by LSF at normoxia and hyperoxia are much lower than the ground truth $s p \mathrm{O}_{2}$ readings. 

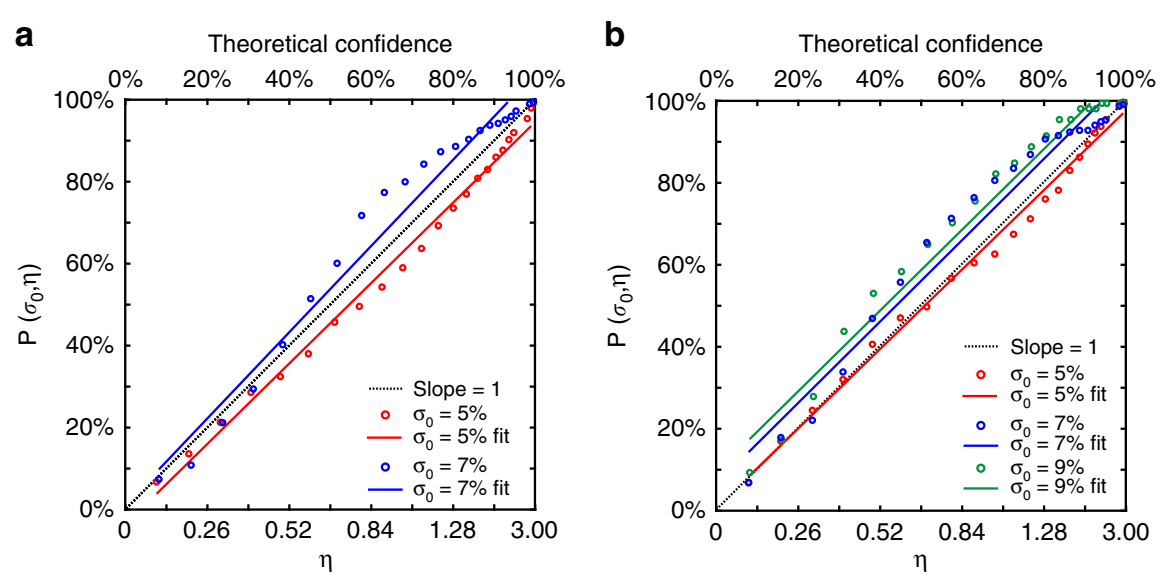

Fig. 5 Statistical analysis of the $\mathrm{sO}_{2}$ predictions with the quantified uncertainty. a The linear fits of $P\left(\sigma_{0}, \eta\right)$ to $\eta$ and the confidence for the FNN model when the uncertainty measured by the standard deviation $\left(\sigma_{0}\right)$ is $5 \%$ and $7 \%$. b The same graph for the CNN model when the standard deviation $\left(\sigma_{0}\right)$ is $5 \%, 7 \%$, and $9 \%$.

Table 1 Parameters of the linear fit of $P\left(\sigma_{0}, \eta\right)$ to theoretical confidence for $\mathrm{SO}_{2}$ predictions with different uncertainties $(\sigma)$ by the FNN and CNN models

\begin{tabular}{|c|c|c|c|}
\hline Models and uncertainties & Slope & Constant & Datasets \\
\hline The FNN model $(\sigma: 5 \% \pm 1 \%)$ & 0.9843 & -0.04286 & 232 \\
\hline The FNN model $(\sigma: 7 \% \pm 1 \%)$ & 1.0551 & 0.01411 & 230 \\
\hline The CNN model $(\sigma: 5 \% \pm 1 \%)$ & 0.9726 & 0.003070 & 185 \\
\hline The CNN model $(\sigma: 7 \% \pm 1 \%)$ & 0.9955 & 0.06697 & 236 \\
\hline The CNN model ( $\sigma: 9 \% \pm 1 \%)$ & 0.9884 & 0.09652 & 75 \\
\hline
\end{tabular}

There are also higher variances in the $s \mathrm{O}_{2}$ results within each individual blood vessel obtained by LSF than by DSL, particularly in Fig. 6g, i. These results clearly indicate the superior robustness and resilience of DSL to variations in experimental conditions and within vessels. Importantly, our DSL models enable direct visualization of the "en face" uncertainty maps of the $s \mathrm{O}_{2}$ predictions in Fig. 7 . The FNN and CNN have similar uncertainty estimations on the $s \mathrm{O}_{2}$ predictions (Fig. $7 \mathrm{a}-\mathrm{f}$ ), consistent with the previous characterization of $\sigma$ at $\sim 5-7 \%$ (Fig. 4). Under hypoxia, the $s \mathrm{O}_{2}$ estimation appears to be inconsistent at the periphery, as indicated by the black arrows in Fig. 6a, $\mathrm{d}$. We attribute this to the extremely poor signal levels at those regions where severe vignetting was present in the raw data (Fig. S5).

\section{Cross validations of the DSL models}

To cross validate our DSL models, we split the training and testing datasets differently and repeated all the analyses for two additional cases. Specifically, Rat 3 in Fig. 3a and Rat 1 in Fig. $3 \mathrm{~b}$ serve as the testing data in the first case, and Rat
1 in Fig. 3a and Rat 5 in Fig. 3b, in the second case. Together with the original testing case (Fig. 4), the MSEs of all the $s \mathrm{O}_{2}$ predictions are summarized in Table S2. In all scenarios, the MSEs from DSL are significantly lower than those from LSF by at least $50 \%$. We also repeated the arterial $s \mathrm{O}_{2}$ testing against the ground truth (Fig. S6-S1) and generated the $s \mathrm{O}_{2}$ and uncertainty maps (Fig. 8S-11S) in both cross-validation cases. All the DSL $s \mathrm{O}_{2}$ maps clearly show the arteriovenous contrasts and the rising $s \mathrm{O}_{2}$ from hypoxia to hyperoxia. All the uncertainty maps also display similar levels of overall confidence.

\section{Discussion}

In this paper, we present a new framework for opticalimaging oximetry based on DSL. The DSL method offers several unique advantages compared to the existing LSF method. First, it bypasses the need for any rigid analytical models and is highly flexible and resilient to experimental variations. We tested DSL on two datasets from two separate vis-OCT experiments and showed that DSL maintained consistent agreement with the ground truth $s p \mathrm{O}_{2}$ despite the many differences between these two experiments. In contrast, an optimized LSF with the identical parametric settings generated significantly worse accuracy. Second, without the restriction of any rigid models, DSL allows more flexible and efficient use of the data. Here, we demonstrate the effectiveness of using the spectra from both the middle and bottom of the vessels, since both carry $s \mathrm{O}_{2}$-dependent spectral contrast. Most importantly, DSL not only provides the point estimate of $s \mathrm{O}_{2}$ but also quantifies the tandem uncertainty of the prediction. Quantifying the statistical uncertainty for each measurement is not possible using the traditional LSF approach; however, it is valuable in assessing the fidelity of each measurement, particularly in clinical applications. We 


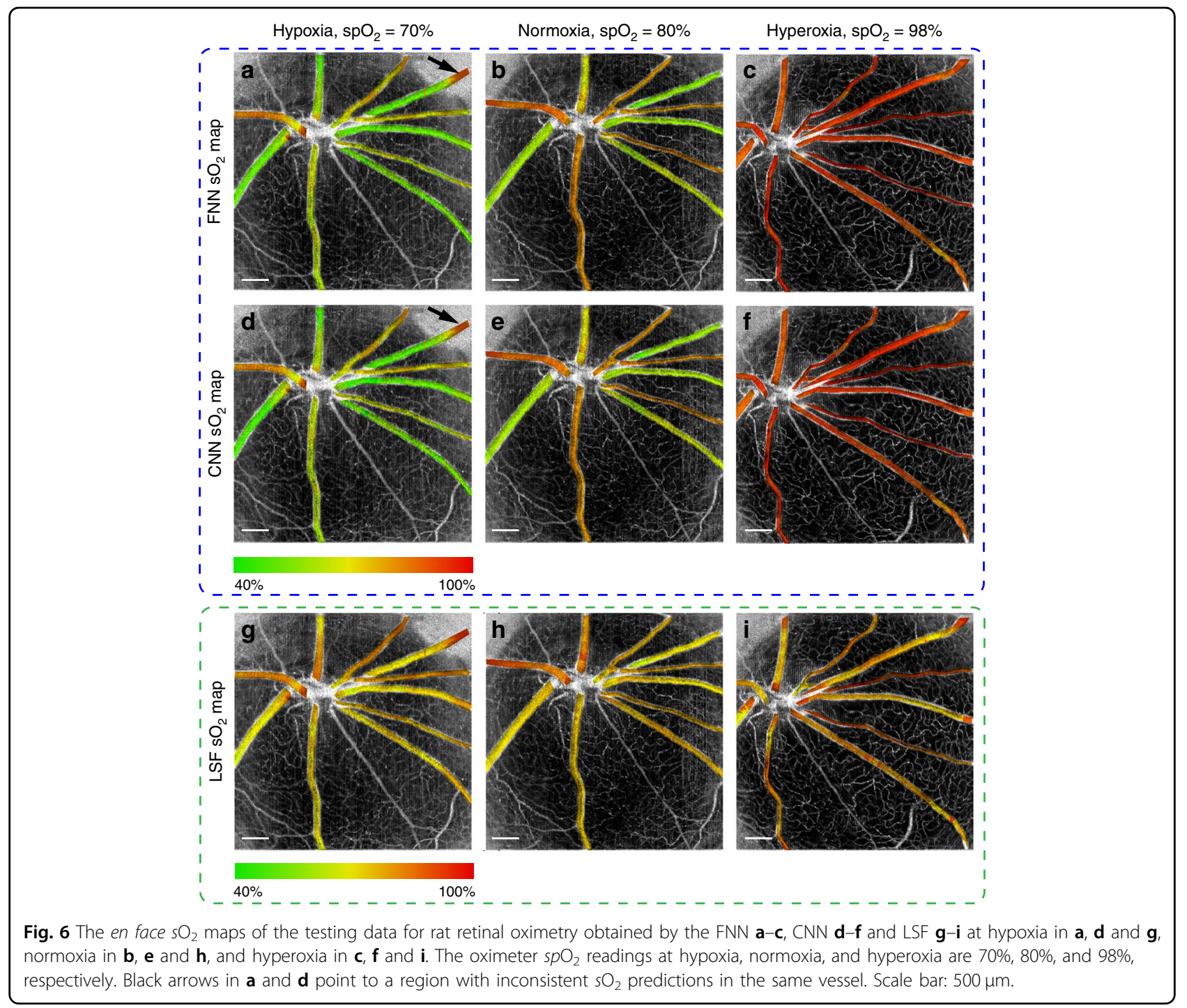

validate the uncertainty quantification by using the reliability diagram, and for the first time to our knowledge, we constructed an uncertainty map of in vivo imaging oximetry showing the estimation confidence by DSL. More generally, our DSL framework presents an attractive data-driven approach for other inverse scattering spectral analyses beyond oximetry.

There are still possible venues to improve the $s \mathrm{O}_{2}$ and uncertainty map predictions. The overall design of our current approach aims to establish a data-driven regression model directly from spectra to $s p \mathrm{O}_{2}$ without considering the spatial information present in the raw OCT data. The benefit of this approach is that a conceptually simple 1D neural network can be trained and tested from randomly shuffled spectral OCT data, in which each spectrum is independent. On the other hand, when we reconstruct the en face maps, the quality of the maps is also subject to spatially dependent data abnormalities, such as low signal contrast, as shown in Fig. 6 in the periphery region. This is a limitation of our current DSL model that may be addressed by a complex $2 \mathrm{D} / 3 \mathrm{D}$ spatiospectral model that incorporates both oxygen maps as the ground truth ${ }^{49}$ and additional spatial information in our future work. Despite this, our DSL prediction performs well and faithfully follows the physiological rules described in the section on en face $s \mathrm{O}_{2}$ maps. In many cases with sufficient signal contrast, uncertainty prediction does provide reasonable confidence assessments, as shown in a few zoomed-in regions in Fig. 8. The full en face maps are provided in Figs. S8-S11. In all these cases, it is shown that the uncertainty levels are higher (i.e., less confidence) in the regions where the $s \mathrm{O}_{2}$ predictions suffer from inconsistency within the vessel, which justifies the utility of the en face uncertainty maps. 

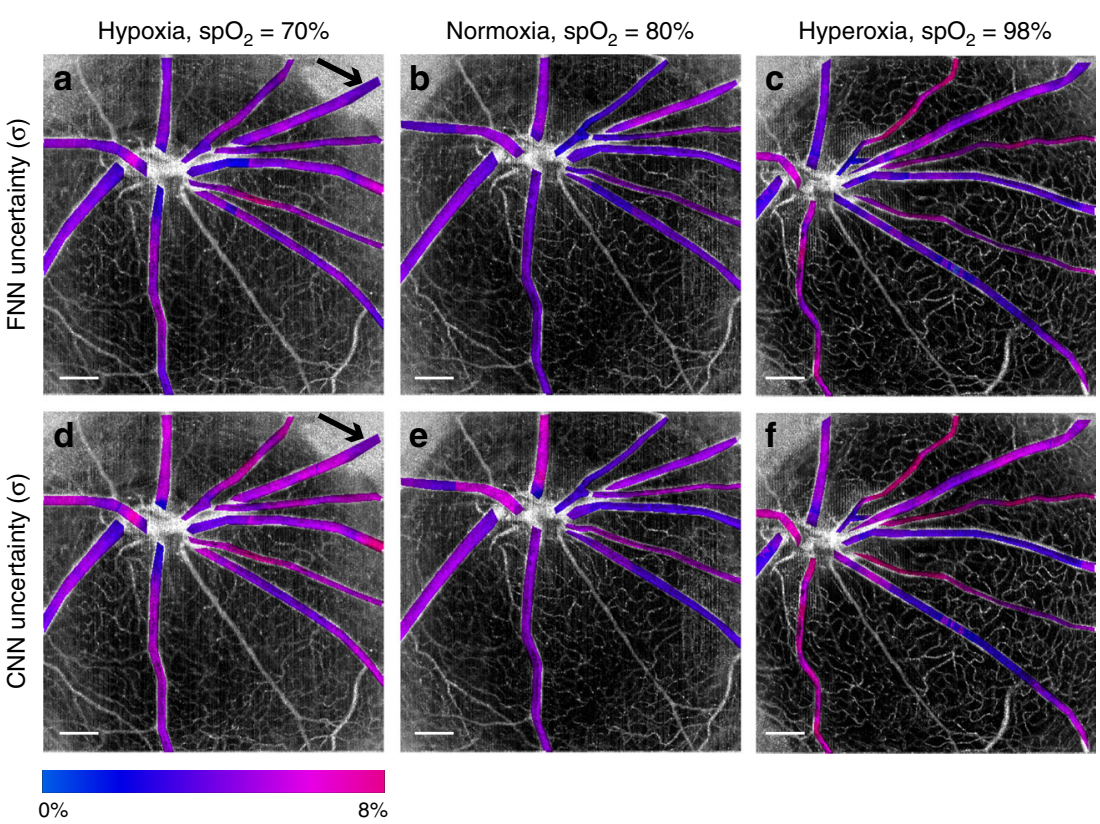

Fig. 7 The en face uncertainty $(\sigma)$ maps for $\mathrm{sO}_{2}$ predictions corresponding to Fig. $\mathbf{6 a}$, $\mathbf{f}$. a-c Uncertainty maps predicted by the $\mathrm{FNN}$ model under three ventilation conditions. $\mathbf{d}-\mathbf{f}$ Uncertainty maps obtained by the CNN model under three ventilation conditions. Black arrows in $\mathbf{a}$ and $\mathbf{d}$ point to a region with inconsistent $\mathrm{sO}_{2}$ predictions in the same vessel. Scale bar: $500 \mu \mathrm{m}$.

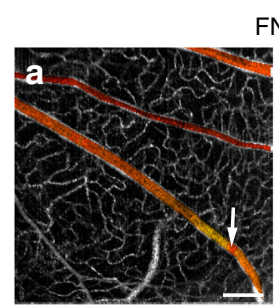

FNN
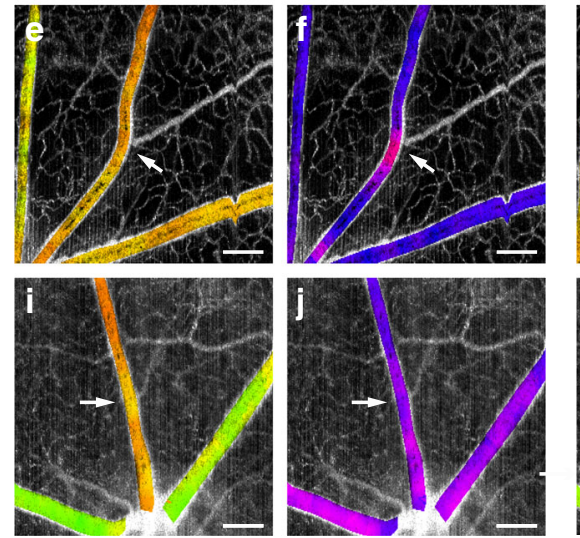

$40 \%$

$\mathrm{SO}_{2}$
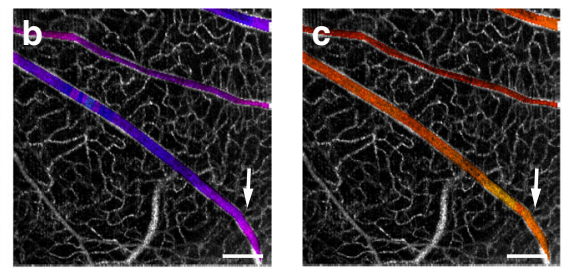

CNN
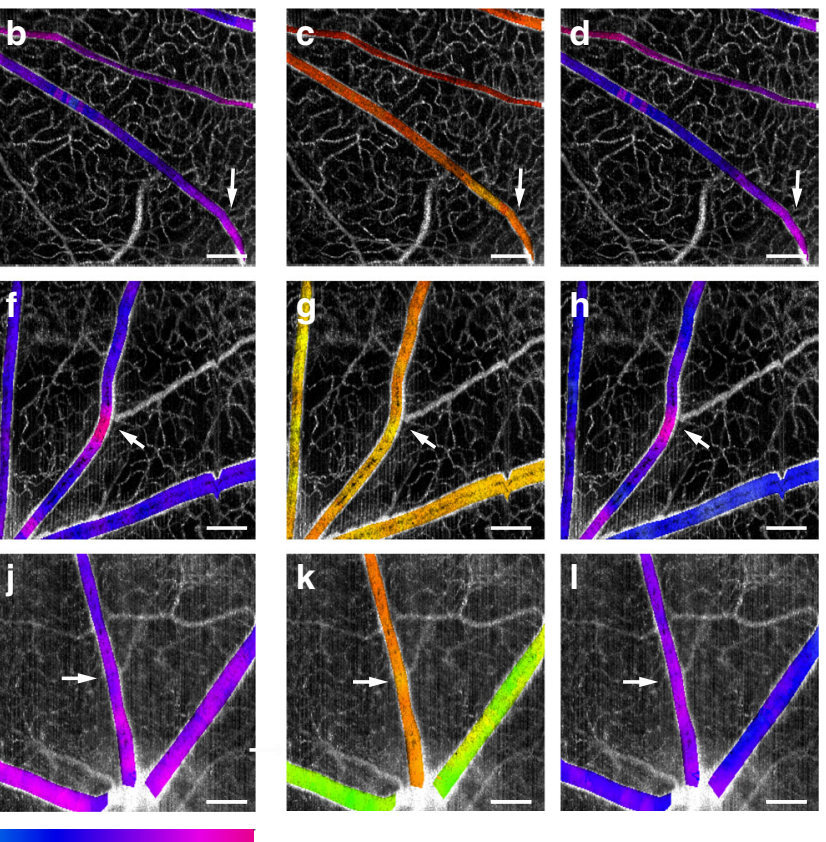

Fig. 8 Zoomed-in views of the $\mathbf{s O}_{\mathbf{2}}$ and the corresponding uncertainty maps. a- $\mathbf{d}$ The $\mathrm{sO}_{2}$ and uncertainty at hyperoxia obtained by the FNN and $\mathrm{CNN}$, respectively, with $\mathrm{spO}_{2}=98 \%$. e- $\mathbf{h}$ The $\mathrm{sO}_{2}$ and uncertainty at normoxia obtained by the $\mathrm{FNN}$ and $\mathrm{CNN}$, respectively, with $s p \mathrm{O}_{2}=76 \%$. i-I The $\mathrm{sO}_{2}$ and uncertainty at normoxia obtained by the FNN and CNN, respectively, with $s p \mathrm{O}_{2}=80 \%$. Scale bar: $500 \mu \mathrm{m}$. Arrows point to the locations where $\mathrm{s}_{2}$ prediction shows inconsistency within a vessel, which are successfully detected by increased uncertainty levels in the corresponding uncertainty map. 
The rationale of using pulse oximetry as the arterial $s \mathrm{O}_{2}$ ground truth is for the ease of clinical translation, since pulse oximetry is a standard-of-care method in clinics. Thus, we expect the same framework to be duplicated in clinical studies. Due to the cardiac cycle, arterial blood has a pulsatile flow pattern, by which pulse oximetry provides consistent and accurate arterial $s p \mathrm{O}_{2}$, regardless of the measurement sites (e.g., at the ear lobe, fingers, foot (in the case of an infant), or skin with the reflectance mode $)^{50-52}$. Therefore, it is convenient and accurate to use $s p \mathrm{O}_{2}$ as a ground truth for $s \mathrm{O}_{2}$ in retinal arteries. For veins, although we do not have the ground truth, our methodology is expected to be valid because the spectroscopic properties of the blood are independent of the arteries or veins but are oxygenation-dependent. The experiments in both datasets changed the oxygen content in the ventilation air, a common approach in physiological studies, to globally modulate the systemic blood oxygenation. By doing so, the network attempts to establish the mapping between the spectral features from the arterial blood and the $s p \mathrm{O}_{2}$. As long as the venous $s \mathrm{O}_{2}$ falls within the ranges of the training dataset, the networks are expected to produce reliable prediction since the network is fed with the spectral data without prior knowledge of their arterial or venous origins.

We envision that the method presented here can generate immediate impacts in ophthalmology, as shown in recent preclinical studies on diabetic retinopathy, glaucoma, and retinal vessel occlusion ${ }^{43-47}$. In particular, since the spectroscopic features of haemoglobin are universal in rodents and humans, we expect the framework developed in this work to be rapidly adapted to human vis-OCT data. Beyond the vis-OCT and retina, we believe the DSL-enhanced optical oximetry may find broad applications on other tissue sites, as long as there are sufficient vascular signal contrast and accessible training datasets. More generally, our DSL framework presents an attractive data-driven approach for other inverse scattering spectral analyses beyond oximetry.

\section{Materials and methods}

\section{Vis-OCT experiments}

The vis-OCT systems in refs. ${ }^{13,15}$ had the same spectral range (from 520 to $630 \mathrm{~nm}$ ), with the same lateral and axial resolutions, estimated to be 15 and $1.7 \mu \mathrm{m}$, respectively. The scanning protocol in ref. ${ }^{13}$ used a raster scan over a $20^{\circ}$ square retinal area covering a field of view (FOV) of $2.51 \mathrm{~mm} \times 2.51 \mathrm{~mm}$, with $256 \times 256$ pixels in each direction at a $25 \mathrm{kHz}$ A-line rate. The exposure time for the spectrometer camera was $37 \mu \mathrm{s}$. The entire vis-OCT image stack took $3.3 \mathrm{~s}$ to acquire. The scanning protocol in ref. ${ }^{15}$ was for optical coherence tomography angiography, scanning a $40^{\circ}$ square retinal area covering an FOV of $4.37 \mathrm{~mm} \times$ $4.37 \mathrm{~mm}$, with 400 pixels in the A-line direction and 512 pixels in the B-scan direction at a $50 \mathrm{kHz}$ A-line rate. The exposure time for the spectrometer camera was $17 \mu \mathrm{s}$. For the sake of vis-OCT angiography, there were repetitive $(5 \times)$ unidirectional B-scans of the same cross section, giving a total of $5 \times 512 \mathrm{~B}$-scans for each acquisition. The entire visOCT image stack took $25.6 \mathrm{~s}$ to acquire.

\section{Spectral extraction and data preprocessing}

Wavelength-dependent vis-OCT images were first generated by a short-time Fourier transform (STFT) with 14 equally spaced Gaussian spectral windows in the $k$-space. The wavelength spans from 523.4 to $604.5 \mathrm{~nm}$. The size (FWHM) of the Gaussian window in the $k$-space is $0.32 \mu^{-1}$, corresponding to a bandwidth of $\sim 17 \mathrm{~nm}$ at $585 \mathrm{~nm}$. After STFT, a spectrum can be obtained at each 3D vis-OCT voxel. Next, we performed segmentation to isolate the spectra within retinal arterioles ${ }^{17,18}$. Retinal blood vessels are first segmented from the en face projection of the $3 \mathrm{D}$ vis-OCT image by a threshold-based algorithm ${ }^{18}$; next, all A-lines within the segmented retinal arterioles are shifted in the axial direction in reference to the retinal surface and randomly shuffled. Here, we applied the rolling average, which analyses data by creating a series of averages of different subsets, on the shuffled A-lines before extracting the input spectra for the DSL models. Specifically, a rolling average of 100 shuffled A-lines in ref. ${ }^{13}$ and 250 shuffled Alines in ref. ${ }^{15}$ was performed with 50 and 125 rolling step sizes, respectively. Because the major vessels are located on top of the retina, the signal within the vessels can be averaged in reference to the retinal surface to generate one spectrum. We located the bottom vessel wall ${ }^{13,25}$ and averaged signals within $\pm 16.6 \mu \mathrm{m}$ to generate the vessel bottom spectrum. We then averaged the signal from $\sim 25 \pm$ 8.31 and $41.6 \pm 8.31 \mu \mathrm{m}$ above the bottom vessel wall to generate the vessel centre and top spectra, respectively. For DSL, the two spectra from the vessel bottom and centre were concatenated as a single spectrum signal. Finally, each individual spectrum input was normalized by the mean of the combined signal from all three spectra (vessel bottom, center, and top) to ensure similar scaling of all datasets before neural network training.

\section{Principle of least-squares fitting}

Vis-OCT uses ballistic photon and coherence gating to localize the optical signal within a tissue volume. At the bottom of the vessel wall, light double-passing through the vessel lumen gives rise to the detectable spectral contrast, which can be analytically formulated based on Beer's law ${ }^{13}$

$$
I\left(s \mathrm{O}_{2} \mid \lambda, z\right)=I_{0}(\lambda) \sqrt{R_{0} r(\lambda)} \mathrm{e}^{-\left[\mathrm{sO}_{2} \times \mu_{\mathrm{HbO}_{2}}(\lambda)+\left(1-\mathrm{sO}_{2}\right) \times \mu_{\mathrm{Hb}}(\lambda)\right] z}
$$

where $I_{0}(\lambda)$ is the spectrum of the light source; $R_{0}$ is the reflectance of the reference arm and assumed to be a 
constant; and $r(\lambda)$ (dimensionless) is the reflectance at the vessel wall, the scattering spectrum of which can be modelled by a power law under the first Born approximation $r(\lambda)=A \lambda^{-\alpha}$, with $A$ being a dimensionless constant and $\alpha$ modelling the decaying scattering spectrum from the vessel wall. The optical attenuation coefficient $\mu$ $\left(\mathrm{mm}^{-1}\right)$ combines the absorption $\left(\mu_{\mathrm{a}}\right)$ and scattering coefficients $\left(\mu_{\mathrm{s}}\right)$ of the whole blood, which are both wavelength- and $\mathrm{sO}_{2}$-dependent:

$$
\mu=\mu_{\mathrm{a}}+W \mu_{\mathrm{s}}
$$

where $W$ is a scaling factor for the scattering coefficient in a range from 0 to $1^{6,17,53}$. The subscripts $\mathrm{Hb}$ and $\mathrm{HbO}_{2}$ denote the contribution from the deoxygenated and oxygenated blood, respectively. $z$ denotes the lightpenetration depth.

To estimate $s \mathrm{O}_{2}$, the traditional approach applies the least-squares procedure that fits the vis-OCT spectral measurement to the analytical model by minimizing the total squares of the error, as illustrated in Fig. 1a:

$$
\min _{s \mathrm{O}_{2}, A, \alpha} \sum_{\lambda}\left\|\log \left[I_{\mathrm{m}}(\lambda)\right]-\log \left[I\left(s \mathrm{O}_{2}, A, \alpha \mid \lambda, z=D\right)\right]\right\|^{2}
$$

where $I_{\mathrm{m}}$ is typically taken as the vis-OCT spectral measurements extracted from the bottom of the vessel to maximize the spectroscopic contrast. The LSF model parameters can be optimized by adjusting the scaling factor $W$ and the spectral wavelength range. We ensured that the whole spectral range covers $\sim 550-585 \mathrm{~nm}$ to include the most dominant spectral contrast between oxygenated and deoxygenated blood. The optimization minimized the MSE between the predicted $s \mathrm{O}_{2}$ and the ground truth $s p \mathrm{O}_{2}$. Figure $\mathrm{S} 12$ shows the MSE with varying $W$ and fitting spectral range for the combined data from refs. ${ }^{13,15}$ and for data from each reference separately. Since the DSL models are trained by a mixture of both data sources, to ensure a fair comparison, we used the optimized LSF model for the combined datasets, where $W=0.12$ and the fitting spectral range is $548-586 \mathrm{~nm}$, respectively (Fig. S12a).

In this analytical model, two free parameters $(A, \alpha)$, in addition to the unknown $s \mathrm{O}_{2}$ level, are introduced to more accurately capture realistic biophysical interactions. However, in practice, these two parameters cannot fully capture all the experimental variabilities. While other models may reduce the free parameters to avoid overfitting $^{14,15,19,20,24,54}$, this approach in general is nonetheless rigid and over-simplified with respect to the actual experiments.

\section{Principle of deep spectral learning}

In DSL, instead of using a rigid analytical model, we train a neural network to link the spectral measurements and the independently measured $s \mathrm{O}_{2}$ labels, as illustrated in Fig. 1b. By doing so, DSL bypasses the need for the parametric tuning and model simplification and approximation needed in fitting the analytical models. Furthermore, by removing the restrictions imposed by the analytical model, DSL allows utilizing multiple sets of spectral measurements taken at different depths and enables a more holistic spectral-s $\mathrm{O}_{2}$ analysis. Specifically, we demonstrate high-quality predictions using concatenated spectra data from both the bottom and the center of the vessels in vis-OCT. Because the pulse oximeter measures the systemic arterial $s \mathrm{O}_{2}$, the same as with the retinal arterioles, we use the retinal arterial spectra as the training input paired with the independently measured pulse oximeter $s \mathrm{O}_{2}\left(s p \mathrm{O}_{2}\right)$ as the ground truth label. After training, the network makes predictions for both arterials and veins.

In addition to $s \mathrm{O}_{2}$ prediction, a major important feature of our data-driven DSL method is to quantify the uncertainty for each prediction. To do so, we specifically design the loss function for training the network to properly capture the underlying statistics of the data. The commonly used loss function, that is, the mean squared error (MSE), assumes a homogeneous Gaussian distribution of the errors in the predictions. This assumption severely limits its ability to adapt different types of spectral data variations (e.g., spectral signal outliers, non-uniform noise, and unevenly sampled data) that are inevitably inhomogeneous. To account for this, we design a customized loss function derived from a heterogeneous Gaussian distribution model. Using the training data set $\left(I_{i},\left[s p \mathrm{O}_{2}\right]_{i}\right), i=1,2, \ldots, N$, where $I_{i}$ and $\left[s p \mathrm{O}_{2}\right]_{i}$ are the $i$ th vis-OCT spectral measurement and the ground truth pulse oximeter $s p \mathrm{O}_{2}$, respectively, our loss function $L_{\mathrm{G}}(w)$ is

$$
L_{\mathrm{G}}(w)=\sum_{i=1}^{N}\left\{\frac{\left(\left[s \mathrm{O}_{2}\right]_{i}(w)-\left[s p \mathrm{O}_{2}\right]_{i}\right)^{2}}{\sigma_{i}^{2}(w)}+\log \left[\sigma_{i}^{2}(w)\right]\right\}
$$

where $\left[s \mathrm{O}_{2}\right]_{i}$ and $\sigma_{i}$ denote the neural network predicted mean and standard deviation, respectively, of the underlying Gaussian distribution for the $i^{\text {th }}$ training data pair; $w$ is the learned neural network weights. The main idea of this loss function assumes that the prediction made on each spectrum follows a distinct Gaussian distribution, and the network is trained to predict the underlying mean and standard deviation ${ }^{55}$. Accordingly, the standard deviation $\sigma$ quantifies the uncertainty for each $s \mathrm{O}_{2}$ prediction. 
We investigate two neural network models, including an FNN and a CNN model, the network architectures of which are shown in Fig. 2a, b, respectively. The detailed descriptions of the network architectures are included in the following section.

\section{Network architectures and training}

The first FNN model, as illustrated in Fig. 2a, concatenates two spectra from both the bottom and centre of the vessels with a size of $1 \times 28$ as the input. The output predicts both the mean $s \mathrm{O}_{2}$ level and the uncertainty (measured by the standard deviation) in two output channels, both of which are single values in units of volume percentage of the blood being oxygenated. The model has two hidden layers, each having 24 units. We use the ReLU-activation function in the two inner layers and the sigmoid activation function in the final layer to normalize the predictions between $0 \%$ and $100 \%$.

The second CNN model, as illustrated in Fig. 2b, takes the same input as the FNN model and predicts the $s \mathrm{O}_{2}$ with uncertainty. The model has two convolutional layers, with each having a filter size of 3 and a filter number of 30 , and one flatten layer. We use the ReLU-activation function in the two convolutional layers and the sigmoid activation function in the final layer.

All data processing and network training are implemented in Python using the TensorFlow/Keras library. Both models were trained with an initial learning rate of $2.5 \times 10^{-3}$, and we gradually decreased the rate by $1 /$ $(1+\alpha N)$, where $N$ is the epoch number and $\alpha$ is a decay rate set to 0.01 . The same total epoch number of 2000 with the same batch size of 50 ensured that the learning curve could reach a plateau. We set the validation split ratio as 0.2 and selected the model with the minimum validation loss as the optimal one for following $s \mathrm{O}_{2}$ prediction.

\section{Loss function for uncertainty quantification}

In the proposed DSL model, denoted by its network weights $w$, the network makes estimation on both the mean and the standard deviation of $s \mathrm{O}_{2}$ given the input spectral measurement. Assuming that the $s \mathrm{O}_{2}$ of all retinal arterioles of a rat at each particular oxygenation status satisfy different Gaussian probability distributions,

$$
p_{w}\left(\mathrm{sO}_{2} \mid I_{i}\right)=N\left(\mathrm{sO}_{2}, \sigma^{2}\right)
$$

where the mean and the standard deviation of the Gaussian distribution are denoted as $s \mathrm{O}_{2}$ and $\sigma$, respectively, and $N$ denotes the normal distribution. The neural network learns a highly complex nonlinear function. During the training, the network weights, $w$, are estimated by maximizing the joint likelihood over $N$ training data pairs:

$$
\begin{aligned}
& w=\arg \max \operatorname{likelihood}(w) \\
& w \in F
\end{aligned}
$$

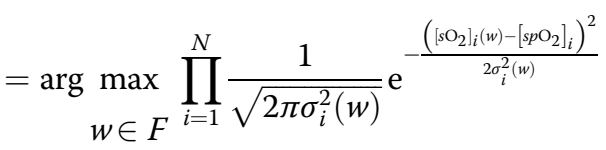

Equivalently, the customized Gaussian loss function $L_{\mathrm{G}}$ $(w)$ is minimized when training the DSL models in Eq. (5).

\section{Reconstruction of en face maps of $\mathrm{sO}_{2}$ and uncertainty $(\sigma)$}

To reconstruct a 2D en face map for $s \mathrm{O}_{2}$ or uncertainty $(\sigma)$, we applied the same spatial signal averaging procedure as described in "Spectral extraction and data preprocessing" section, but this averaging was done pixelwisely within each blood vessel. We first segmented the en face vessel area manually. For each pixel from the 2D en face map within the segmented blood vessel, an arteriole or a venule, its depth-dependent spectra were generated by averaging spectral signals from its 100 (in the first literature) or 250 (in the second literature) nearest neighbours based on the Euclidian distance. Then, these spectra would be input into the FNN or CNN model to predict a $s \mathrm{O}_{2}$ with uncertainty for this pixel. Next, the above two steps would be iterated pixel-wisely until all pixels within this particular blood vessel had predicted $s \mathrm{O}_{2}$ and uncertainty. Finally, the above three steps would be iterated until all arterioles and venules of the rat retina had predicted $s \mathrm{O}_{2}$ and uncertainty for displaying their $2 \mathrm{D}$ en face maps. To generate Figs. 6 and 7, we applied an algorithm based on the HSV (hue, saturation, and value) colour model, where the predicted $s \mathrm{O}_{2}$ or uncertainty corresponds to the image hue, and the angiography signal intensity corresponds to the image value and saturation.

\footnotetext{
Acknowledgements

We acknowledge Siyu Chen for providing the original data in his vis-OCT experiment. We acknowledge Robert Linsenmeier and Hao F. Zhang for their support in this study, and both of the original vis-OCT studies. We

acknowledge Vadim Backman for Rongrong Liu's role in this study. Research is funded by National Science Foundation (1813848); National Institute of Health (R01CA224911, R01CA232015, R01NS108464, R21EY029412); Bright Focus

Foundation (G2017077, M2018132).
}

\section{Funding}

National Science Foundation (1813848); National Institute of Health (R01CA224911, R01CA232015, R01NS108464, R21EY029412); Bright Focus Foundation (G2017077, M2018132).

\footnotetext{
Author details

${ }^{1}$ Department of Biomedical Engineering, Northwestern University, Evanston, IL 60208, USA. ²Department of Electrical and Computer Engineering, Boston University, Boston, MA 02215, USA. ${ }^{3}$ Department of Biomedical Engineering, Boston University, Boston, MA 02215, USA. ${ }^{4}$ Department of Medicine, Boston University School of Medicine, Boston Medical Center, Boston, MA 02118, USA
} 


\section{Author contributions}

R.L. established the structure of the two deep spectral learning models and trained them to predict retinal vascular $\mathrm{sO}_{2}$ with confidence quantification. S.C. and L.T. helped with the statistical theory and codes for confidence quantification. The idea originated from a discussion between J.Y. and R.L, and J.Y. and L.T. directed and supervised the project. All authors discussed the results and contributed to the manuscript.

\section{Conflict of interest}

The authors declare that they have no conflict of interest.

Supplementary information is available for this paper at https://doi.org/ 10.1038/s41377-019-0216-0.

Received: 8 July 2019 Revised: 17 October 2019 Accepted: 1 November 2019

Published online: 20 November 2019

\section{References}

1. Stefánsson, E. Ocular oxygenation and the treatment of diabetic retinopathy. Sun. Ophthalmol. 51, 364-380 (2006).

2. Vajkoczy, P., Ullrich, A. \& Menger, M. D. Intravital fluorescence videomicroscopy to study tumor angiogenesis and microcirculation. Neoplasia 2, 53-61 (2002).

3. Levy, B. I. et al. Impaired tissue perfu37sion: a pathology common to hypertension, obesity, and diabetes mellitus. Circulation 118, 968-976 (2008).

4. Edwards-Richards, A. et al. Capillary rarefaction: an early marker of microvascular disease in young hemodialysis patients. Clin. Kidney J. 7, 569-574 (2014).

5. Kalaria, R. N. Neurodegenerative disease: diabetes, microvascular pathology and Alzheimer disease. Nat. Rev. Neurol. Vol. 5, 305-306 (2009).

6. Faber, D. J. et al. Oxygen saturation dependent absorption and scattering of whole blood. Proceedings Volume 5316, Coherence Domain Optical Methods and Optical Coherence Tomography in Biomedicine VIII. San Jose, CA, United States: SPIE, 2004

7. Nguyen, J. Q. M. et al. Spatial frequency domain imaging of burn wounds in a preclinical49 model of graded burn severity. J. Biomed. Opt. 18, 066010 (2013).

8. Gioux, S. et al. First-in-human pilot study of a spatial frequency domain oxygenation imaging system. J. Biomed. Opt. 16, 086015 (2011).

9. Delori, F. C. Noninvasive technique for oximetry of blood in retinal vessels. Appl. Opt. 27, 1113-1125 (1988).

10. Palsson, $\mathrm{O}$. et al. Retinal oximetry images must be standardized: a methodological analysis. Investig. Ophthalmol. Vis. Sci. 53, 1729-1733 (2012).

11. Blair, N. P. et al. Retinal oximetry and vessel diameter measurements with a commercially available scanning laser ophthalmoscope in diabetic retinopathy. Investig. Ophthalmol. Vis. Sci. 58, 5556-5563 (2017).

12. Kristjansdottir, J. V. et al. Retinal oximetry with a scanning laser ophthalmoscope. Investig. Ophthalmol. Vis. Sci. 55, 3120-3126 (2014).

13. $\mathrm{Yi}$, J. et al. Visible light optical coherence tomography measures retinal oxygen metabolic response to systemic oxygenation. Light.: Sci. Appl. Vol. 4, e334 (2015).

14. Shu, X. et al. Visible-light optical coherence tomography: a review. J. Biomed. Opt. 22, 121707 (2017).

15. Chen, S. Y., Yi, J. \& Zhang, H. F. Measuring oxygen saturation in retinal and choroidal circulations in rats using visible light optical coherence tomography angiography. Biomed. Opt. Express 6, 2840-2853 (2015).

16. Liu, R. R. et al. Theoretical model for optical oximetry at the capillary level: exploring hemoglobin oxygen saturation through backscattering of single red blood cells. J. Biomed. Opt. 22, 025002 (2017).

17. Liu, R. et al. Single capillary oximetry and tissue ultrastructural sensing by dualband dual-scan inverse spectroscopic optical coherence tomography. Light.: Sci. Appl. Vol. 7, 57 (2018).

18. Liu, R. R. et al. Quantitative quality-control metrics for in vivo oximetry in small vessels by visible light optical coherence tomography angiography. Biomed. Opt. Express 10, 465-486 (2019).

19. Pi, S. H. et al. Rodent retinal circulation organization and oxygen metabolism revealed by visible-light optical coherence tomography. Biomed. Opt. Express 9, 5851-5862 (2018).
20. $\mathrm{Pi}, \mathrm{S}$. H. et al. Automated spectroscopic retinal oximetry with visible-light optical coherence tomography. Biomed. Opt. Express 9, 2056-2067 (2018).

21. Soetikno, B. T. et al. Visible-light optical coherence tomography oximetry based on circumpapillary scan and graph-search segmentation. Biomed. Opt. Express 9, 3640-3652 (2018).

22. Robles, F. E. et al. Molecular imaging true-colour spectroscopic optical coherence tomography. Nat. Photonics 5, 744-747 (2011).

23. Yi, J. \& Li, X. Estimation of oxygen saturation from erythrocytes by highresolution spectroscopic optical coherence tomography. Opt. Lett. 35 2094-2096 (2010).

24. Yi, J. et al. In vivo functional microangiography by visible-light optical coherence tomography. Biomed. Opt. Express 5, 3603-3612 (2014).

25. Yi, J. et al. Visible-light optical coherence tomography for retinal oximetry. Opt. Lett. 38, 1796-1798 (2013).

26. Tan, Y. H. et al. Surface area and pore size characteristics of nanoporous gold subjected to thermal, mechanical, or surface modification studied using gas adsorption isotherms, cyclic voltammetry, thermogravimetric analysis, and scanning electron microscopy. J. Mater. Chem. 22, 6733-6745 (2012).

27. Wang, L. D., Maslov, K. \& Wang, L. V. Single-cell label-free photoacoustic flowoxigraphy in vivo. Proc. Natl Acad. Sci. USA 110, 5759-5764 (2013).

28. Kuranov, R. V. et al. Depth-resolved blood oxygen saturation measurement by dual-wavelength photothermal (DWP) optical coherence tomography. Biomed. Opt. Express 2, 491-504 (2011).

29. Kuranov, R. V. et al. In vivo depth-resolved oxygen saturation by dualwavelength photothermal (DWP) OCT. Opt. Express 19, 23831-23844 (2011).

30. Cox, B. T. et al. Quantitative spectroscopic photoacoustic imaging: a review. J. Biomed. Opt. 17, 061202 (2012).

31. Yao, J. J. et al. High-speed label-free functional photoacoustic microscopy of mouse brain in action. Nat. Methods 12, 407-410 (2015).

32. Sinha, A. et al. Lensless computational imaging through deep learning. Optica 4, 1117-1125 (2017)

33. Li, Y. Z., Xue, Y. \& Tian, L. Deep speckle correlation: a deep learning approach toward scalable imaging through scattering media. Optica 5, 1181-1190 (2018).

34. Yichen, W. C. et al. Extended depth-of-field in holographic imaging using deep-learning-based autofocusing and phase recovery. Optica 5, 704-710 (2018).

35. Liu, X. M. et al. Semi-supervised automatic segmentation of layer and fluid region in retinal optical coherence tomography images using adversarial learning. IEEE Access 7, 3046-3061 (2019).

36. Xue, Y. J. et al. Reliable deep-learning-based phase imaging with uncertainty quantification. Optica 6, 618-629 (2019).

37. Ching, T. et al. Opportunities and obstacles for deep learning in biology and medicine. J. R. Soc. Interface 15, 20170387 (2018).

38. Hammer, M. et al. Retinal vessel oximetry-calibration, compensation for vessel diameter and fundus pigmentation, and reproducibility. J. Biomed. Opt. 13, 054015 (2008).

39. Yi, J. et al. Human retinal imaging using visible-light optical coherence tomography guided by scanning laser ophthalmoscopy. Biomed. Opt. Express 6. 3701-3713 (2015)

40. Zhang, T. W., Kho, A. M. \& Srinivasan, V. J. Improving visible light OCT of the human retina with rapid spectral shaping and axial tracking. Biomed. Opt. Express 10, 2918-2931 (2019).

41. Ju, M. J. et al. Visible light sensorless adaptive optics for retinal structure and fluorescence imaging. Opt. Lett. 43, 5162-5165 (2018).

42. Chen, S. Y. et al. Retinal oximetry in humans using visible-light optical coherence tomography. Biomed. Opt. Express 8, 1415-1429 (2017).

43. Liu, W. et al. Increased retinal oxygen metabolism precedes microvascular alterations in type 1 diabetic mice. Investig. Ophthalmol. Vis. Sci. 58, 981-989 (2017).

44. Soetikno, B. T. et al. Inner retinal oxygen metabolism in the 50/10 oxygeninduced retinopathy model. Sci. Rep. 5, 16752 (2015).

45. Song, W. Y. et al. Longitudinal detection of retinal alterations by visible and near-infrared optical coherence tomography in a dexamethasone-induced ocular hypertension mouse model. Neurophotonics 6, 041103 (2019).

46. $\mathrm{Pi}, \mathrm{S}$. H. et al. Monitoring retinal responses to acute intraocular pressure elevation in rats with visible light optical coherence tomography. Neurophotonics 6, 041104 (2019).

47. Soetikno, B. T. et al. Optical coherence tomography angiography of retinal vascular occlusions produced by imaging-guided laser photocoagulation. Biomed. Opt. Express 8, 3571-3582 (2017). 
48. Weigert, M. et al. Content-aware image restoration: pushing the limits of fluorescence microscopy. Nat. Methods 15, 1090-1097 (2018).

49. Sencan, I. et al. Two-photon phosphorescence lifetime microscopy of retinal capillary plexus oxygenation in mice. J. Biomed. Opt. 23, 126501 (2018).

50. Webster, J. G. Design of Pulse Oximeters (CRC Press, Bristol, 1997).

51. Nakajimi, S. et al. New pulsed-type earpiece oximeter (author's transl). Kokyu Junkan 23, 709-713 (1975).

52. Khan, Y. et al. A flexible organic reflectance oximeter array. Proc. Natl Acad. Sci. USA 115, E11015-E11024 (2018).
53. Bosschaart, N. et al. A literature review and novel theoretical approach on the optical properties of whole blood. Lasers Med. Sci. 29, 453-479 (2014).

54. Chong, S. P. et al. Cerebral metabolic rate of oxygen $\left(\mathrm{CMRO}_{2}\right)$ assessed by combined Doppler and spectroscopic OCT. Biomed. Opt. Express 6, 3941-3951 (2015).

55. Kendall, A. \& Gal, Y. What uncertainties do we need in bayesian deep learning for computer vision? (eds. H., Wallach, H., Larochelle, A., Beygelzimer, F., d'Alché-Buc, E., Fox. \& R., Garnett.) In Proc. 30th Advances Neural Information Processing Systems (NIPS). 5580-5590, The Neural Information Processing Systems Foundation: 2017 